\title{
Treinta años de traducciones literarias del italiano en México
}

\author{
Rodrigo JARDÓN HERRERA \\ Universidad Nacional Autónoma de México \\ Sabina LONGHITANO PIAZZA \\ Universidad Nacional Autónoma de México
}

\begin{abstract}
Este artículo presenta un panorama y algunas reflexiones críticas sobre la difusión editorial de la literatura y la cultura italianas en México en los últimos treinta años, relacionándolos con el crecimiento y la difusión de los estudios de italianística en la Facultad de Filosofía y Letras de la UNAM y enfocándose en las figuras de traductores y en los programas editoriales más relevantes, para sentar las bases de una reflexión sobre la recepción de la literatura italiana, tanto canónica como contemporánea, en el México actual.
\end{abstract}

PALABRAS CLAVE: literatura italiana, cultura italiana, traductores literarios italiano-español, editoriales, México.

In this paper we present an overview and some critical reflexions on the editorial diffusion of italian literature and culture in Mexico over the last thirty years, related to the growth and spread of italian studies in the Faculty of Philosophy and Literature of the UNAM, focusing on the most relevant figures of translators and publishers, in order to start a reflection about the reception of both canonic and contemporary italian literature in Mexico nowadays.

KEYWORDS: italian studies, italian-spanish literary translators, publishers, Mexico.

La literatura italiana ha ejercido desde siempre una sana y vigorosa influencia sobre la literatura escrita en español [...]. La relación entre nuestras lenguas y nuestras culturas ha sido de diálogo constante y se ha mostrado siempre liberal, rica y estimulante a lo largo ya de varios siglos. Por ello es de celebrarse la instauración de la Cátedra Extraordinaria Italo Calvino en esta nuestra Facultad. Es muy significativo que se haya bautizado con ese nombre ya que Calvino ha ejercido gran influencia sobre la literatura universal y en particular a la narrativa escrita en español.

Hernán Lara Zavala, 2005: 21.

El objetivo de este artículo es hacer un recuento de la difusión editorial de la literatura italiana en México en los últimos treinta años, haciendo hincapié en su relación tanto con el Departamento de Letras Italianas de nuestra Facultad como con la Cátedra 
Extraordinaria Italo Calvino, instituida en la Facultad de Filosofía y Letras de la Universidad Nacional Autónoma de México en 1994 con la contribución de la Secretaría de Relaciones Exteriores (Ministero degli Affari Esteri) de Italia, con el objetivo de promover los estudios de italianística en México. ${ }^{1}$

Las literaturas necesitan entablar diálogo y las traducciones son una evidencia del mismo. Muchos autores, como es el caso de Calvino, forman parte del horizonte cultural de nuestros intelectuales gracias a las traducciones; es por esta razón que nos interesa reflexionar sobre los traductores que en nuestro país han trabajado para crear nuevos horizontes y espacios de diálogo intercultural.

Es innegable que la mayor parte de la cultura italiana se ha dado a conocer en México gracias a las traducciones que se realizaban en otros países de Latinoamérica, específicamente en Argentina (Adriana Hidalgo Editora o El Cuenco de la Plata son ejemplos recientes), mas sobre todo por lo que se importaba de España. Las grandes editoriales ibéricas (Seix-Barral, Alfaguara, Tusquets, Anagrama, Siruela, Acantilado, Lumen, etcétera) dominan desde hace casi cincuenta años la industria del libro en español, y sus publicaciones han definido el impacto de algunos escritores en el mundo hispánico. Nuestras bibliotecas cuentan con un importante acervo de literaturas europeas; sin embargo, el precio de los libros, elevado debido a los costos de importación, y el predominio de la literatura anglófona han sido factores que dificultan la difusión de otras literaturas. Las editoriales locales y los traductores nacionales juegan un papel decisivo; por ello vale la pena empezar con una reflexión sobre la recepción de la literatura italiana en nuestro contexto.

La historia de la traducción del italiano en México es un fenómeno que se ha estudiado muy poco. Ciertamente no es posible enfrentar en un solo artículo un análisis exhaustivo de la relación entre la cultura italiana y la mexicana; es por eso que en este artículo decidimos hacer un recuento detallado, aunque seguramente no exhaustivo, de las traducciones literarias de los últimos treinta años. Hay que observar que la institución de la Cátedra Extraordinaria Italo Calvino y el crecimiento tanto de la matrícula como de la cantidad de titulados del Departamento de Letras Italianas coinciden con el periodo más productivo de la traducción del italiano en nuestro país ya que, de los años noventa a la fecha, han surgido nuevos traductores y se han publicados trabajos notables.

Antes de empezar nuestro recuento de las últimas décadas, podemos afirmar que entre el siglo XIX y el XX se realizaron importantes traducciones del italiano en México, pero se trataba de iniciativas aisladas, no de proyectos orgánicos dedicados a la difusión

\footnotetext{
${ }^{1}$ El objetivo de los congresos internacionales organizados desde 1995 con cadencia bianual por la Cátedra Extraordinaria Italo Calvino es el de reunir a italianistas de todo el mundo para compartir los resultados de sus investigaciones, contribuir a la difusión de la literatura y cultura italianas e impulsar los estudios de italianística en México. Los resultados de cada congreso se materializan en la publicación de un libro que reúne una selección dictaminada de artículos relativos a las ponencias presentadas. Los tres cuadernos y los doce libros publicados hasta la fecha por la UNAM y la Cátedra Extraordinaria Italo Calvino (un treceavo libro monográfico, dedicado a Giovanni Boccaccio, fue publicado en Florencia por Franco Cesati) se encuentran disponibles en el Repositorio Institucional de nuestra Facultad: <http://ru.ffyl.unam.mx/handle/10391/31>.
} 
de la cultura italiana. Los estudios sobre este diálogo cultural han sido encabezados, en el Departamento de Letras Italianas, por Fernando Ibarra, quien específicamente ha rastreado, en revistas y periódicos del siglo XIX de nuestro país, las traducciones y la crítica literaria sobre Italia; por otro lado, una de nuestras estudiantes, Xrysw Susana Marroquín Caravantes, se tituló recientemente con una tesis que aborda la misma temática durante el Porfiriato: Difusión y traducción de literatura italiana en México (puente entre los siglos XIX Y XX).

Puesto que en este artículo solamente nos abocamos a los últimos treinta años, decidimos organizar de forma no cronológica la información, recabada de las páginas web de las editoriales y de las bibliotecas a nuestra disposición. Consideramos preferible organizar nuestro discurso a partir de las características más relevantes tanto de los traductores como de las colecciones específicas de las editoriales mexicanas.

Comencemos por la UNAM, que es donde más traducciones del italiano se han publicado. En las colecciones Bitácora Poética y Cuadernos del Seminario de Poética, del Instituto de Investigaciones Filológicas, Annunziata Rossi ha conjuntado el estudio de la literatura italiana y su traducción; además de ser una especialista de la literatura del siglo XX, en particular de su relación con el fascismo, Rossi ha contribuido bastante a los estudios sobre el Renacimiento. El primer renacimiento florentino. Ideas y presagios del descubrimiento de América (1998) y Ensayos sobre el Renacimiento italiano (2002) son recopilaciones de ensayos que se relacionan directamente con el análisis y la selección de su antología de cuentos del Renacimiento El relato del Renacimiento italiano (1996; segunda edición, bilingüe, 2007) que ha permitido dar a conocer, por primera vez en México, los textos de los cuales el teatro europeo, sobre todo Shakespeare, tomó las tramas de gran parte de sus obras.

La decana de nuestro departamento y fundadora de la Cátedra Extraordinaria Italo Calvino, Mariapia Lamberti, dantista y profesora de literatura italiana, tradujo otro texto clave de ese periodo para una editorial independiente: El cuento del Gordo carpintero, atribuido a Antonio di Tuccio Manetti (Taller Ditoría, 2008). Junto con José Luis Bernal, quien por muchos años fue uno de nuestros profesores de literatura y de traducción, Lamberti tradujo el tratado de Marsilio Ficino Sobre el amor. Comentarios al Banquete de Platón (UNAM, 1994); ${ }^{2}$ como parte de una amplia investigación de Olga Sáenz, del Instituto de Investigaciones Estéticas de la UNAM, Bernal y Lamberti también tradujeron una vasta selección de manifiestos futurista para el libro El futurismo italiano (2010). Para la colección Material de Lectura, la más difundida de la UNAM y que está ahora completamente digitalizada, ${ }^{3}$ Lamberti y Bernal publicaron también una antología de los Cantos de Giacomo Leopardi (traducción de José Luis Bernal ${ }^{4}$ e introducción de Mariapia Lamberti, 2012).

\footnotetext{
${ }^{2}$ Si bien la versión original de tratado ficiniano está en latín, el mismo autor hizo de ella un volgarizzamento, es decir, una traducción al vulgar florentino, que es lo que Lamberti y Bernal traducen.

${ }^{3}<\mathrm{http}$ ://www.materialdelectura.unam.mx>.

${ }^{4}$ La traducción completa de esta obra fue publicada por la editorial española La Veleta, en 1998.
} 
Durante los más de veinte años de vida de la Cátedra Extraordinaria Italo Calvino es posible notar una profunda conexión entre los temas estudiados por nuestros colegas y la literatura italiana que poco a poco ha ganado terreno entre el público mexicano. Entre los muchos libros al cuidado de Mariapia Lamberti para la UNAM, El narrador y el crítico (2004) es fundamental. Se trata de la primera antología que ofrece un panorama de textos dedicados al rico diálogo que escritores y críticos entablaron en el siglo XX. Por nombrar solamente una de sus mayores contribuciones, en este libro se encuentra la única traducción al español de Giacomo Debenedetti: un fragmento de un ensayo sobre la obra de Luigi Pirandello proveniente de Il romanzo del Novecento, y el ensayo, publicado en Intermezzo, dedicado a Dino Buzzati; ambas traducciones fueron realizadas por Guillermo Fernández, sobre quien nos detendremos más adelante. Gracias a esta antología fue posible dar a conocer en nuestro ámbito académico una de las líneas fundamentales de la literatura italiana del siglo pasado: la crítica literaria, que, más allá de ser un vehículo para la interpretación, también se constituye como una actividad eminentemente creativa. Como escribió Debenedetti refiriéndose a Renato Serra: "lo strumento del critico è lo stile del critico"5 (2003: 670). Finalmente es importante mencionar la traducción de José Luis Bernal y la edición de Mariapia Lamberti de cuatro lecciones impartidas por Alfonso Berardinelli como profesor invitado por la Cátedra Extraordinaria Italo Calvino, que la UNAM publicó en 1996, con el título 50 anni di letteratura italiana / 50 años de literatura italiana (Lezioni / Lecciones 1945-1995) como el primero de los Cuadernos de la Cátedra Extraordinaria Italo Calvino.

Fabio Morábito, quien es investigador en el Centro de Estudios de Poética del Instituto de Investigaciones Filológicas de la UNAM y profesor de traducción en nuestro Departamento, en 1995 publicó para la UNAM Los pastores sin ovejas, un ensayo que retoma el tema de su tesis de licenciatura sobre la academia poética Arcadia del siglo XVIII y su impronta en la literatura europea. Como él mismo afirma, este trabajo se nutrió de las clases de Annunziata Rossi, Mariapia Lamberti y Alaíde Foppa, y lo llevó a realizar una estancia de investigación con Cesare Segre en Italia, percatándose de que Aminta de Torquato Tasso desde 1604 no había sido traducido nuevamente al español. En su introducción Morábito explica la pertinencia de volver a traducir la obra: la versión de 1604, a cargo del poeta Juan de Jaúregui, respondía a una época en la que el traductor se sentía en derecho de modificar libremente el texto original, en este caso aumentando más de cien versos en diferentes partes de la obra. Como parte de ese estudio introductorio, Morábito aventura una definición sobre el oficio de traductor que vale la pena citar:

[...] el original es como un río de aguas turbulentas y, si no somos capaces de crear una mínima presa de captación de esas aguas que nos permita tratar con ellas bajo ciertas condiciones impuestas por nosotros, probablemente el río nos aniquilaría. Ser fiel al río tal como es, es imposible, porque el río lo estamos siempre interpretando, o

\footnotetext{
5 "El instrumento del crítico es el estilo del crítico" (traducción de los autores).
} 
sea inventando. Hay que redondear esa invención cuando traducimos, crear una segunda fidelidad, más íntima y vaga que la que nos exige la correspondencia idiomática, y responder siempre dentro de ella. En suma, hay que adherirse a una imagen vital concreta, a una postura y a algún tipo de máscara, pues traducir, finalmente, es interpretar, e interpretar es actuar, en el sentido teatral del término (2001: 26).

En 2001 su traducción del Aminta se publicó, por iniciativa de Augusto Monterroso, en la colección de la UNAM Nuestros Clásicos. La traducción de Morábito de la poesía completa de Eugenio Montale (2006) para la editorial española Galaxia Gutemberg es el resultado de sus estudios de Maestría, en los que realizó un análisis de la obra del poeta genovés y una traducción comentada del primer poemario, Huesos de sepia. La última de sus traducciones relacionadas con su investigación es una antología de la poeta Patrizia Cavalli: Yo casi siempre duermo (2008), que apareció en la colección de la UNAM, El Puente.

Fernando Ibarra, uno de nuestros expertos en literatura medieval, coordinó y curó la traducción, realizada por un equipo de estudiantes avanzados de traducción, de las Rimas de Giovanni Boccaccio, que está en espera de publicación por parte de la UNAM.

Sabina Longhitano, experta en literatura del Renacimiento, tradujo, para una edición conmemorativa facsimilar del Príncipe de Machiavelli (Taurus y Senado de la República, 2013), la célebre carta del autor a Francesco Vettori del 10 de diciembre 1513, la nota introductoria del primer editor, Antonio Blado D'Asola y el prólogo de MaurizioViroli. ${ }^{6}$ Longhitano tradujo también algunas citas del latín y del griego en la edición de De la disimulación honesta di Torquato Accetto (Colección Libros de Artefacto, 2001) traducida por Rossella Bergamaschi, académica de la Universidad Autónoma Metropolitana.

Nuestro traductor y experto en poesía del Novecento, Stefano Strazzabosco, quien tradujo La miel de Tonino Guerra (Ediciones Sin Nombre, 2004) y estuvo a cargo, junto con Marco Antonio Campos, de una antología poética del siglo Xx, Veintidós poetas italianos (UNAM, 2006). Cabe mencionar que Campos publicó en 1979 para la UNAM La alegría de Ungaretti. Strazzabosco y Campos también colaboraron para publicar en la editorial El Tucán de Virginia, que edita exclusivamente poesía, Vendrá la muerte y tendrá tus ojos de Cesare Pavese (2016). Cabe también mencionar que Strazzabosco coordinó también la traducción, a cargo de un equipo de estudiantes de nuestro Departamento, de la antología de poetas contemporáneos sicilianos Sicilia: la tierra del titiritero, publicada por una editorial independiente (Circo Literario, 2017).

Éstos son ejemplos de los proyectos que el Departamento de Letras Italianas busca promover: trabajos en los que la investigación y la traducción del área de italianística vayan de la mano, y que involucren la colaboración tanto de académicos como de estudiantes y tesistas de licenciatura y de posgrado.

\footnotetext{
${ }^{6}$ Para la traducción del Príncipe, en esta edición se compraron los derechos de la traducción hecha por Eleanor Leonetti para Espasa Calpe.
} 
Otros dos egresados de letras italianas, Rodrigo Jardón Herrera y Diego Antonio Mejía Estévez, publicaron para la UNAM una antología de ensayos de Italo Svevo, $L a$ corrupción del alma, en la colección Pequeños Grandes Ensayos (2016), y Bestias de Federigo Tozzi para la colección Licenciado Vidriera (2017).

Destaca la labor de una de nuestras colegas: Clara Ferri. Es la primera de nuestras profesoras de traducción que en sus clases conjugó de forma consistente los estudios teorícos y la práctica traductiva. Su propuesta didáctica se caracteriza por abordar textos que no se limitan al ámbito de la literatura, y que, por lo tanto, permiten que los alumnos amplíen su conocimiento del oficio de la traducción para futuras oportunidades laborales. Gracias a su laboratorio de subtitulaje de materiales audiovisuales, el único de nuestra Facultad, Clara Ferri toca problemáticas particulares de la lengua y la cultura italiana que no podrían formar parte del resto de nuestros cursos de licenciatura y que, sin duda, son fundamentales, tomando en cuenta que en nuestro país solamente en la UNAM se puede estudiar a nivel universitario la literatura y la cultura italiana. Sus traducciones al español incluyen a autores como Norberto Bobbio, Antonio Tabucchi, Angelod'Orsi, Carlo Ginzburg, Massimo Rizzante y Chiara Gamberale. En el campo de los materiales audiovisuales su trayectoria incluye más de cincuenta películas y documentales. Estos trabajos han permitido dar a conocer tanto cuestiones nodales de la cultura italiana en México, como el caso de Franco Basaglia, como difundir en Italia problemáticas sociales de nuestro país, como es el caso de la desaparición forzada de cuarenta y tres estudiantes de la Escuela Normal Rural de Ayotzinapa, en septiembre de 2014. Actualmente, Ferri está terminando una nueva traducción de la serie de libros de Emilio Salgari dedicada a los piratas del Caribe para los tipos de Conaculta: El Corsario Negro, La reina de los Caribes, Yolanda la hija del Corsario Negro, El hijo del Corsario Rojo y Los últimos filibusteros, que fueron publicados en 2018.

En 2008 Italia fue el país invitado en la Feria Internacional del Libro de Guadalajara y una de las revistas literarias de la Universidad de Guadalajara, Luvina, dedicó el número 53 (invierno 2008), ${ }^{7}$ titulado Stazione Italia, a una amplia y variada selección de autores contemporáneos italianos, muchos de los cuales eran completamente desconocidos en México. En sus páginas confluyen distintas generaciones y sus voces se entrelazan para presentar un innovador mosaico que da cuenta de la heterogeneidad de la prosa y la poesía que caracteriza la producción literaria italiana desde el siglo pasado hasta la fecha: a pesar de la falta de una justificación, por parte del editor, se trata de la más ambiciosa antología de literatura italiana realizada en nuestro país. En ella participaron muchos de los traductores mexicanos más representativos del periodo que abarcamos, pero también un gran número de nuestros estudiantes avanzados o recién egresados del área de traducción. A continuación incluimos el índice completo de los textos y los traductores, como una forma de recuperar y valorizar las huellas de este intenso trabajo:

\footnotetext{
${ }^{7}$ Disponible en $<$ https://luvina.com.mx/foros/index.php?option=com_content\&task=section\&id=9\&I temid $=41>$.
} 
Mariapia Lamberti, Seis dimensiones de la poesía italiana actual de Alfonso Berardinelli; María Teresa Meneses, Fráncfort. Harem de libros de Giorgio Manganelli, Muchos saludos de Antonio Tabucchi, La pluma de Tommaso Landolfi, Una visita dificil (4 de junio de 1943) de Dino Buzzati, Rap y poesía de Eduardo Sanguineti, Revolución y literatura de Giuseppe Tomasi di Lampedusa, Por un cigarro de Mario Praz; Jorge Alberto Aguayo, West coast, September eleven de Alessandro Baricco; Brenda Mora, Antes de desaparecer (fragmento) de Mauro Covacich, Todo, todo junto de Gian Mario Villalta; Guillermo Fernández, El alumno de Giovanni Turra, La misma raza de Fabrizio Bernini, Visto una camiseta de Paolo Ruffilli, Se entra en una casa ajena de Matteo Zattoni, Mundo: sé de Andrea Zanzotto, Locura y El manicomio de Alda Merini; Martha Canfield, Caía ya la noche, Mirando los restos de un casete en la pausa de un viaje de verano y De un paisaje de Milena Barberis visto a través del 'pulpo'de Apollinaire de Valerio Magrelli, El mundo subterráneo de Athanasius Kircher de Claudio Magris, Vuelve el viento frío de Alessio Brandolini, Magritte de Carlo Bordini y La travesía de Maurizio Cucchi; Beatriz Galindo, Algo dulce de Paolo Lagazzi; Fabio Morábito, Cada día hermoso y Caigo y vuelvo a caer de Patrizia Cavalli; Sergio Trejo, Dónde está la esterilidad de Dario Bellezza; Mara Donat y Marco Antonio Campos, Para la ventana nueva de Andrea Zanzotto; Jeannette L. Clariond, Postguerra de Fabio Ciriachi, Sangre de Arnaldo Ederle, No hay cosa y Días de Silvia Bre, Ya nada hay de Anna Maria Carpi, El corazón pesado de Italo Testa; Renato Sandoval Bacigalupo, Modorra de Franco Marcoaldi, Tema del adiós de Milo de Angelis, III (De la superación) y Cuando el tiempo de Giovanna Frene, Las historias están de Stefano Raimondi, Postal y Amantes de Salvatore Ritrovato, Cómo llamamos, Dios y Tanto me molesta de Franco Loi, Roma (I y II) de Franco Buffoni; Jeamel Frolres-Haboud, Tiresias de Giuliano Mesa, Criterio de los cristales de Marco Giovenale; Manuela Torquati, Surtidor de abrazos de Daniela Tomerini; Juan Castro, Como un eco de Jolanda Insana, Imagina de Giancarlo Pontiggia, Visión de un espectro y Malos cambios de Antonella Anedda; Silvia Cruz, Julio, el último vuelo de Alberto Garin, Los libros son como los árboles: aproximaciones a la narrativa italiana contemporánea de Stefano Strazzabosco; Marcela Tavera Soria, El futbolista de Bilbao de Dacia Maraini, Trenes de Bianca Garavelli; Dulce María Zúñiga, Bien de Umberto Fiori, El viento en el mar es un ser de escamas predispuesto a la masacre de Maria Grazia Calandrone; finalmente, la traducción que el poeta italiano Emilio Cocco hizo de su propio poema Monte Celano.

El blog STACI (Seminario de Traducción de Textos de Autores Contemporáneos Italianos $),{ }^{8}$ creado y coordinado por Clara Ferri, forma parte de la página web de la Facultad de Filosofía y Letras de la UNAM y ofrece la posibilidad de aproximarse a traducciones muy meditadas. En este espacio periódicamente se publican fragmentos, en edición bilingüe, de traducciones realizadas por nuestros estudiantes. Con el paso del tiempo, se ha convertido en un observatorio de facto sobre la literatura y el pensa-

\footnotetext{
${ }^{8}<$ http://staci.filos.unam.mx $>$.
} 
miento italiano contemporáneo: se pueden encontrar autores ya clásicos, como Leonardo Sciascia, Pier Vittorio Tondelli y Remo Bodei, y otros que gracias a esta iniciativa ya están a disposición de un público más amplio, como Stefano Benni, Carlo Lucarelli, Barbara Garlaschelli y Mauro Corona. Los traductores forman parte de diferentes generaciones de nuestra Licenciatura en Letras Italianas. Sus nombres son: María Teresa Aguilar Garrido, Edita Diana Cabrera Becerra, Silvia Cruz López, Mariana Isabel Enríquez Alatriste, Claudia Isabel Flores Ramírez, Nadia Daniela García Medina, Violeta Rosales Hernández, Karol Einhorn López Aldana, Brenda Olimpia Mora Santana, Jaime Rea González, Carolina Rodríguez Monti, Mauricio Horacio Ronquillo Valdez, Margarita Liliana Salvatierra Cruz, Lourdes Estela Sánchez Durán, Juan Diego Tapia Perea, Marcos Tavera Casado y Néstor Josué David Venegas Ramos.

A la par de esta iniciativa, Clara Ferri editó recientemente, junto al investigador y periodista Fabrizio Lorusso, Ni una más (Universidad Iberoamericana de Nuevo León, 2017), una antología de cuentos de autores italianos, traducidos tanto por miembros de STACI como por otros estudiantes y egresados del área de traducción, que analizan críticamente la problemática del feminicidio, un tema dolorosamente presente en todo el mundo, que involucra dramáticamente a Italia y al contexto mexicano y latinoamericano actual.

El Periódico de Poesía, que a partir de 2007 dejó su versión impresa para ser una publicación exclusivamente digital, es otro de los espacios de la UNAM en la que están presentes traducciones de poetas italianos. Entre los trabajos más recientes están las traducciones de Diego Tapia, Jesús y la lagartija, El hombre de arcilla, La hormiga blanca y Tiempos modernos de Claudio Pagelli; ${ }^{9}$ XLIX, De la imagen tensa y Viático de Clemente Rebora; ${ }^{10}$ de Eleonora González Capria, las rimas 32, 124 y 311 de Gaspara Stampa; ${ }^{11}$ de Reinhard Huamán Mori, Aquel lejos de nosotros de Milo de Angelis. ${ }^{12}$ En 2016, el estudiante de letras italianas Rafael Hernández Aguilar ganó el premio del Círculo de Traductores del Periódico de Poesía, con la traducción de Mitos, una breve antologia de Carla de Bellis. ${ }^{13}$

Es dentro de nuestra Universidad y gracias a nuestros egresados y académicos donde se ha publicado el mayor número de traducciones del italiano. En la colección Material de Lectura, Hugo Gutierrez Vega publicó una antología de poetas italianos (Cardarelli, Montale, Pasolini, Pavese, Quasimodo, Saba y Ungaretti, 2007), y Edmundo Valadés una selección de cuentos de Massimo Bontempelli (2008). Entre las publicaciones del CUEC, Miguel Bustos García tradujo todos los ensayos de Empirismo erético que Pier Paolo Pasolini dedicó al cine: Cinema. El cine como semiología de la realidad (2006). Finalmente, en una de las antologías del Centro de Enseñanza de Lenguas

\footnotetext{
${ }^{9}$ Periódico de Poesía 92, septiembre 2016. <http://www.periodicodepoesia.unam.mx/index.php/ traducciones/63-traducciones/4333-092-traducciones-claudio-pagelli>.

${ }^{10}$ Periódico de Poesía, 94, noviembre 2016. <http://www.periodicodepoesia.unam.mx/index.php/ traducciones/63-traducciones/4421-no-094-traducciones-clemente-rebora>.

${ }^{11}$ Periódico de Poesía, 97, marzo 2017. <http://www.periodicodepoesia.unam.mx/index.php/4504>.

${ }^{12}$ Periódico de Poesía, 98, abril 2017. <http://www.periodicodepoesia.unam.mx/index.php/4575>.

$13<$ http://www.periodicodepoesia.unam.mx/images/stories/pdf/concurso-1x1-italiano-resultados.pdf $>$.
} 
Extranjeras (Serrano Coronado y Desmet, 2011) fue publicado el cuento "Sámana", del escritor Pino Cacucci, que tradujeron en conjunto Consuelo Aguirre de los Reyes, Patricia Ángeles Delgado, Luciana Minerbi di Segni y Juan Porras Pulido. Cabe mencionar que Tomás Serrano Coronado, profesor de traducción tanto en CELE como en El Colegio de México, tradujo para Joaquín Mortiz El polvo de México de Pino Cacucci (1996) y coordinó también la traducción colectiva de Demasiado corazón, del mismo autor, publicada por el Instituto Italiano de Cultura en 2016.

Lamentablemente la mayor parte de esta ardua producción no logra difundirse a nivel nacional e internacional, porque uno de los problemas más graves de nuestra institución es la falta de un editor general, que también se encargue de la distribución de todos los libros realizados en la UNAM. Cada colección depende del centro de estudios o facultad que la auspició y la distribución no es, a decir verdad, la mejor. Los libros producidos por las instituciones públicas llegan a las bibliotecas públicas del país, pero los lectores no los conocen.

En 2015 Benjamín Mayer Foulkes editó para 17, Instituto de Estudios Críticos, Itinerarios de la cultura contemporánea en México, que contenía las conferencias de un congreso en el que varios intelectuales mexicanos se reunieron a discutir sobre el estado actual de la cultura en nuestro país: gran parte de los debates giró en torno al mundo de la edición. El poeta Luigi Amara argumenta que el problema general de las editoriales universitarias en México es "especialmente desconcertante, porque entiende el libro como un trámite, es cumplir con una obligación al punto de que cuando ya está editado puede meterse en una bodega y se acabó; ya se publicó, ya se ejerció un presupuesto, se hizo lo que se tenía que hacer desde el aparato burocrático" (2015: 69).

Por esta razón, uno de los retos para el futuro consiste en crear espacios adecuados para difundir las obras que ya han sido traducidas y las que se realicen más adelante, porque, como apuntó en ese mismo congreso el investigador de El Colegio de México Fernando Escalante Gonzalbo, estos esfuerzos representan un uso ético de los recursos públicos. No valorar lo que se produce al interior de las universidades públicas equivale a contribuir a la actual crisis institucional que genera proyectos neoliberales de privatización de todos los recursos de un país. Una de las ideas básicas en el proceso de privatización es que el Estado es ineficiente, que es un mal empresario y que las empresas deben hacerse cargo. Sin embargo, la economista italiana Mariana Mazzucato muestra que la mayor creatividad, productividad y rentabilidad se ha producido desde siempre en empresas públicas (2013: passim). Aceptar un subsidio o buscar una forma de cofinanciamiento es perfectamente aceptable con una forma de organización en que también es prioritaria la transparencia. Hay instituciones públicas enormemente creativas, interesantes, productivas y rentables. Las del Estado no son formas necesariamente parasitarias ni corruptas: puede haber muchas fórmulas y habría que pensarlas (Escalante Gonzalbo, 2015: 83).

Pasemos ahora a reseñar a otros traductores mexicanos especializados en la literatura italiana. Tedi López Mills reunió en un volumen, publicado por el Fondo de Cultura Económica, una antología de traducciones realizadas por escritores mexicanos. 
Este ambicioso proyecto presenta un rico panorama de treinta y tres poetas traductores que va de José Emilio Pacheco a Alfonso D'Aquino: Traslaciones. Poetas traductores 1939-1959 (2011). Los poetas que traducen del italiano no son la mayoría, y aquí los queremos enlistar, junto con los poemas que aparecen en la selección de Mills, porque sus elecciones dan cuenta de una parte de la recepción de la literatura italiana en México durante las últimas décadas: Homero Aridjis, El cántico de las creaturas de San Francesco de Assisi y varios fragmentos del Inferno dantesco; Elsa Cros, La balada del exilio de Guido Cavalcanti; Marco Antonio Campos, Desde la cuesta, Cuando se abría el velario, Mujer, Ulises y Tenía de Umberto Saba, Abandono, Cruel adiós, Liguria, Noche de Liguria, Parábola, A la tierra, Paso nocturno y Un fanal de Vincenzo Cardarelli, Soy una criatura, Peregrinaje, San Martín del Carso, Nostalgia, Despedida, Otra noche y Vagabundo de Giuseppe Ungaretti; David Huerta, Taccion i boschi de Torquato Tasso; Francisco Serrano, Mil veces, oh dulce mía guerrera y Si con verme me mata, o si sonrie de Francesco Petrarca; José Luis Rivas, La cabra de Umberto Saba; Fabio Morábito, dieciocho poemas tomados de su traducción de Eugenio Montale, publicada en España; Francisco Segovia, No encuentro paz, ni puedo hacer la guerra de Francesco Petrarca, También tú eres la colina y Eres la tierra y la muerte de Cesare Pavese; Alfonso D’Aquino, El infinito de Giacomo Leopardi, Mañana, Recuerdo de África, Alegría de náufragos, Sereno y Soy una criatura de Giuseppe Ungaretti, Hacia el fondo de Eugenio Montale, Invierno y Hambre de Alfonso Gatto.

Lo que salta a la vista es que Mills no incluyó en su antología al poeta mexicano que se dedicó con mayor pasión a la literatura italiana: Guillermo Fernández.

De todos los traductores no egresados de la UNAM, Fernández es quien más se nutrió de la colaboración con nuestra institución. Sin contar sus traducciones de los años setenta y ochenta, destacan Poesía completa (UnAm, 1991) de Cesare Pavese; Alfabeto pirandelliano (Ediciones El Milagro, 1997) de Leonardo Sciascia, y Poesía y no poesía (UNAM, 1998) de Benedetto Croce, los últimos dos con agudas introducciones de Annunziata Rossi. En la colección Nuestros Clásicos aparecieron obras de gran relevancia para el canon literario de Italia: Cuentos para un año (1993) de Luigi Pirandello; Vida de Benvenuto Cellini, florentino, escrita por él mismo (1995) de Benvenuto Cellini; El cortesano (1997) de Baldassar Castiglione, y Los prometidos (1997) de Alessandro Manzoni. En esa misma colección, Fernández también publicó una versión de El Decamerón (2003) de Giovanni Boccaccio, en la que colaboró VittoreBartra. Para Material de Lectura, Fernández publicó un sinfín de antologías de poesía y narrativa. Los autores que eligió forman un amplio abanico, cuya difusión conforma lo que en nuestro país se conoce como literatura italiana moderna y contemporánea: Eugenio Montale, Mario Luzi, Cesare Pavese, Michelangelo Coviello, Milo de Angelis, Valerio Magrelli, Gino Scartaghiande, Alberto Savinio, Pier Paolo Pasolini, Alberto Moravia, Vitaliano Brancati, Giuseppe Tomasi Lampedusa, Leonardo Sciascia, Dino Buzzati, Italo Calvino y Giovanni Verga. Por último, cabe resaltar que en la colección Poemas y Ensayos, en la que fue publicado Poesía y no poesía de Croce, Fernández 
realizó las únicas antologías de ensayos de crítica literaria y cultural de Montale y de Ungaretti con los que cuenta nuestra tradición bibliográfica. ${ }^{14}$

Fernández se ocupó también, desde mediados de los años noventa hasta 2012, año de su muerte, de la columna La colmena en Italia, dedicada a la traducción de autores italianos principalmente poetas, en la revista La Colmena de la Universidad Autónoma del Estado de México. Es imposible aquí hacer un recuento de todos los autores traducidos en casi veinte años: ${ }^{15}$ además de los autores ya citados, destacan Michelangelo Buonarroti, Francesco Guicciardini, Giovanni Papini, Umberto Saba, Elio Vittorini, Vittorio Sereni, Sandro Penna, Edoardo Sanguineti, Alda Merini, Antonia Pozzi, Patrizia Valduga, Giovanna Frene, Andrea Zanzotto, Antonio Tabucchi, Paolo Ruffilli, Bartolo Cattafi, Michele de Giacomo, Leonardo Sinisgalli, Stefano Strazzabosco y Marco Perilli.

La Universidad Veracruzana cuenta con un ambicioso programa de difusión literaria. En su colección Biblioteca Universitaria, que cuenta con un amplio catálogo de clásicos de la literatura universal, están dos de las obras maestras de la narrativa del siglo Xx: La conciencia de Zeno (2008) de Italo Svevo y El difunto Mattia Pascal (2012) de Luigi Pirandello, pero en estos dos casos, lamentablemente, el traductor no está especificado. En la colección dedicada a reunir las traducciones de uno de sus profesores eméritos y emblemático exponente de la literatura mexicana de la segunda mitad del siglo xx, Sergio Pitol, se encuentra su única traducción del italiano: Salto mortal de Luigi Malerba. En 2016 Ana Villada, una de las estudiantes de Pitol, publicó por esta misma universidad una antología de narrativa: Cuentos del Novecento italiano: un modelo para armar. Esperamos que esta institución siga promoviendo la difusión de la cultura italiana.

Dentro de un proyecto editorial conjunto entre la Universidad Iberoamericana y la editorial Artes de México, destaca la figura del traductor Ernesto Bustos, de origen cubano, residente en México desde 1992 y colaborador de la revista Vuelta, quien tradujo de Eugenio Montale Cuaderno de cuatro años (1999) y de Andrea Zanzotto, una antología poética, titulada Del paisaje al idioma (1996). En México, Bustos colaboró también con editoriales independientes, con la traducción de los Motetes de Eugenio Montale (El Dorado Ediciones, 1997); de Andrea Zanzotto (Para que) (crezca). Ensayos (2012), y de Valerio Magrelli La vicevida (2015), ambas publicadas por Mangos de Hacha. En España, Bustos tradujo Venus herida de Paolo Maurensig (Barcelona, Mondadori, 2000) y Ejercicios de tiptología de Valerio Magrelli (Pre-Textos, Valencia, 2011).

Para seguir el hilo de los proyectos editoriales conjuntos entre universidades y editores, cabe destacar también la actividad del poeta y traductor Emilio Coco, quien en México estuvo a cargo de una antología de Poesía italiana contemporánea, publicada

\footnotetext{
${ }^{14}$ Una bibliografía parcial de las antologías publicadas por Fernández se encuentra en $<$ http://www.literatura.bellasartes.gob.mx/jalisco/4412-fernandez-guillermo.html>.

${ }^{15}$ En el repositorio institucional de la UAEM, la búsqueda "Guillermo Fernández" arroja casi cien resultados: $<$ http://ri.uaemex.mx/discover?scope=/\&rpp=10\&page=10\&query= $\% 22$ Guillermo + Fern $\% \mathrm{C} 3 \% \mathrm{~A} 1 \mathrm{n}$ dez $\% 22 \&$ group_by $=$ none \&etal $=0>$.
} 
en 2010 por La Cabra, con el apoyo del Fondo Nacional para la Cultura y las Artes y de la Universidad Autónoma de Nuevo León. Coco ha publicado numerosas antologías de poetas italianos modernos y contemporáneos tanto en España como en otros países de América Latina.

Una figura fundamental para la difusión de la cultura —en particular histórica, filosófica y antropológica - no sólo italiana sino también lusófona, francófona y anglófona es la traductora uruguaya Stella Mastrangelo, quien ha realizado trabajos que enriquecen mucho el panorama de la cultura italiana en México y Latinoamérica. Gracias a sus traducciones para el Fondo de Cultura Económica se han difundido en América Latina las ideas políticas de figuras tan importantes como Norberto Bobbio (Perfil ideológico del siglo XX en Italia, 1989) y Federico Chabod (La idea de nación, 1987); el historiador Salvatore Lupo (Historia de la mafia: desde sus orígenes hasta nuestos días, 2009), y obras literarias como Crucigrama (1990) de Leonardo Sciascia, y México (1989) de Emilio Cecchi. Para la editorial barcelonesa Acantilado tradujo El universo de los griegos de Oddone Longo (2009). Para nosotros, sus trabajos más relevantes son sus traducciones de Nicolás Maquiavelo: en la colección Clásicos del Pensamiento Político de la Universidad Autónoma de la Ciudad de México se publicó, en edición bilingüe, El príncipe (2008), y para el Fondo de Cultura Económica el Epistolario (1512-1527) del escritor florentino (1990).

En el Fondo de Cultura Económica se encuentra la única traducción del italiano realizada por el filósofo Alejandro Rossi, quien en 1992 dio a conocer una de las obras más queridas de Italo Calvino: Historia de la astronomía de Giorgio Abetti. Dentro de un proyecto de difusión de la literatura infantil y juvenil del Fondo de Cultura Económica, Bruno Aceves tradujo Voces griegas, de Beatrice Masini (2002). Como parte del mismo proyecto editorial, Fabio Morábito ha participado con Pinocho con botas (1992) y La historia y la gloria y otros relatos (1992) de Luigi Malerba; Las aventuras de Pierino en el mercado de Luino (1993) de Piero Chiara, y El planeta de los ratonejos de Renata Schiavo Campo (1992).

Para la editorial mexicana Vuelta, Morábito publicó un texto singular de Tommaso Landolfi, La biere du pecheur (1991; cabe mencionar que el título original es el mismo, está escrito todo en mayúsculas y deliberadamente sin acentos, para simular la enseña de una cervecería).

Pasemos ahora a reseñar lo que se publicó en el programa de difusión cultural que se desarrolló en Conaculta. En esta institución se creó una colección única en su tipo, Cien del Mundo, que incluía obras muy variadas, en términos tanto temporales como de las tradiciones que abarcaba. En ésta era posible acceder a textos que podían ir del Corán a los ensayos literarios de Ezra Pound. En el caso de la literatura italiana las traducciones fueron obras de suma relevancia. Guillermo Fernández tradujo una selección amplia de los artículos periodísticos de Pier Paolo Pasolini que se reunieron en Descripciones de descripciones (1995). Stella Mastrangelo dio a conocer una de las novelas nodales del Romanticismo italiano, Las últimas cartas de Jacopo Ortis (2000) de Ugo Foscolo. Finalmente, también se publicaron, gracias al trabajo de Sergio 
Martínez, dos comedias del último Premio Nobel italiano, Dario Fo: El papa y la bruja. El diablo con tetas (2012). Conaculta comisionó a Clara Ferri una nueva traducción de la serie de libros de Emilio Salgari dedicada a los piratas del Caribe, que salió en 2018.

En el programa editorial que actualmente se desarrolla en la Secretaría de Cultura se creó una colección bastante similar a la de Cien del Mundo: Clásicos para Hoy. La única obra italiana que ha aparecido hasta el momento en ésta es Los novios de Alessandro Manzoni (México, Conaculta, 2014). En la contraportada del libro escriben los editores: "Se ofrece aquí la extraordinaria traducción castellana de Juan Nicasio Gallego editada en Barcelona entre 1836 y 1837, fresca y seductora, contemporánea de la obra original, basada en la edición italiana de 1827'. Esta decisión nos parece muy discutible. Se trata de un fenómeno característico de nuestra historia editorial: comprar derechos de traducciones ya existentes o publicar aquellas que se encuentren libres de derechos. Si consideramos el hecho de que ya contábamos con una versión de esta novela gracias a Fernández y que contamos con un buen número de traductores excelentes, la decisión de los editores nos deja perplejos. Si bien la de Gallego es una traducción interesante por su valor histórico, no creemos que sea el vehículo ideal para un proyecto que se define a sí mismo como pensado para la sociedad actual. El proceso de esa novela es uno de los más complejos que ha tenido la historia literaria italiana: con sólo revisar la edición de 1971 de Lanfranco Caretti es evidente que hubo cambios fundamentales por parte del escritor lombardo en sus ediciones, la que realizó entre 1825 y 1827 y la definitiva de 1840. Estas cuestiones, por otra parte, sí están contempladas en la edición de la UNAM de la novela manzoniana.

Este tipo de casos es bastante frecuente en nuestro contexto. Una pesquisa rápida del catálogo de la biblioteca pública José Vasconcelos, inaugurada en 2006, muestra estas tendencias: la casi totalidad de las obras de la literatura italiana con las que cuenta son ediciones españolas y muchas de las obras publicadas por editoriales mexicanas tiene traducciones compradas de otros países y los dos autores con más obras en las estanterías son viejas traducciones hechas en España de Edmondo de Amicis y Emilio Salgari. Estos datos muestran los obstáculos actuales, pero también los retos a futuro.

En conclusión, los últimos treinta años han sido el periodo más fecundo de la traducción del italiano en nuestro país. Además del trabajo realizado dentro de nuestra institución, han aparecido otros trabajos notables. Rogelio Villarreal y Mauricio Salvador explican que fue a penas al inicio de los años noventa del siglo pasado que nuestro país tuvo un surgimiento muy amplio de editoriales independientes. Ambos académicos argumentan que se trató de un cambio sano para nuestro entorno cultural. Si bien, es cierto que el gobierno dio apoyo a proyectos fundamentales, como la revista Vuelta de Octavio Paz, el control excesivo de las publicaciones en general impedía generar espacios de debate: "publicaciones muy necesarias que daban gran parte de un gran espectro de la cultura mexicana, pero dedicadas a los grandes nombres, a las grandes firmas que todos conocemos. Había otra gente, no necesariamente jóvenes, que querían ser leídos" (Villarreal, 2015: 203). 
A partir de los años cercanos al cambio de milenio se vive una gran efervescencia creativa que se verá reflejada en los catálogos de las editoriales independientes. Esa nueva apertura del mercado, en gran medida nutrida de las nuevas traducciones, contribuyó al aumento de la crítica y, como argumenta Mauricio Salvador, al nacimiento de nuevos horizontes interpretativos: "Los lectores somos las personas más bondadosas que existen: así como nos sorprendemos cada vez, también nos dejamos engañar una y otra vez. Por eso es importante que existan espacios de crítica" (2015: 213). La presencia de la literatura italiana actualmente se puede inscribir en esta búsqueda de vías y espacios de diálogo por parte de intelectuales, académicos, traductores y escritores.

La editorial Sexto Piso tiene una impronta totalmente italiana: uno de sus fundadores, Eduardo Rabasa, ha declarado de inspirarse en - y emular- la función que Roberto Calasso juega en la editorial milanesa Adelphi (2015: 218). De las muchas iniciativas culturales surgidas en los últimos años, Sexto Piso resalta por la elección de sus libros. Rabasa explica que "si el sello es coherente, fiel a su identidad, los lectores confían en el sello y en el editor. El editor hace una especie de curaduría literaria, hace recomendaciones de todo lo que se está publicando. Algunos de nuestros autores son absolutamente desconocidos, pero el peso del resto del catálogo los arropa, de modo que el lector puede decidir leerlos por la confianza que le tiene a la editorial" (2015: 219).

Su catálogo incluye obras fundamentales de Carlo Emilio Gadda, Paolo Volponi y Giorgio Colli, pero sólo enlistaremos las traducciones hechas en México, que son pertinentes para nuestra investigación: Stella Mastrangelo, El patrón de Goffredo Parise (2014); Guillermo Fernández, Aquiles enamorado de Alberto Savinio (2004); Teresa Ramírez Vadillo, El loco impuro (2003) y La locura que viene de las ninfas (2008) de Roberto Calasso; Fernando Macotela, Fellini. Les cuento de mí (2007) de Costanzo Costantini; María Teresa Meneses, Literatura y derecho ante la ley (2009) y El conde y otros relatos (2014) de Claudio Magris. Meneses tradujo también una recopilación de textos periodísticos de Magris, El tallo entre las piedras, para la editorial mexicana Cal y Arena (2007). Volviendo a Sexto Piso, Rossella Bergamaschi y Antonio Castilla tradujeron La persuasión y la retórica de Carlo Michelstaedter (2009), y Ernesto Kavi La muchacha indecible. Mito y misterio en Kore (2014) y El fuego y el relato (2016) de Giorgio Agamben.

En el campo del pensamiento político, la editorial Era se nutrió de la colaboración con la investigadora griego-mexicana Dora Kanoussi para la edición, por primera vez al español, de la edición seminal de Valentino Gerratana, de 1975, de Los cuadernos de la cárcel (2001) de Antonio Gramsci, traducido por Ana María Palos. ${ }^{16}$ Dos años después se publicó el famoso epistolario, Cartas desde la cárcel (2003), siguiendo la edición de Antonio Santucci, con la traducción de Cristina Ortega Kanoussi. Se trata de una contribución fundamental a la difusión de uno de los pensadores italianos que más ha influido en América Latina.

\footnotetext{
${ }^{16}$ Todas las traducciones anteriores de Gramsci, tanto mexicanas como argentinas, se basaban en la edición de Palmiro Togliatti (1948-1951).
} 
Queremos concluir nuestro discurso reseñando la labor de algunas editoriales independientes. El breve y selecto catálogo de la editorial Ai Trani, dirigida por el académico italiano Fabrizio Cossalter, quien por algunos años impartió clases de literatura italiana del siglo XX en nuestro Departamento, publicó textos que se colocan en la encrucijada entre crítica y literatura, como Examen de conciencia de un literato, de Renato Serra (2015), en la traducción a cuatro manos del mismo Cossalter y de Rodrigo Jardón Herrera; dos obras del poeta, narrador, crítico literario y traductor Massimo Rizzante, No somos los últimos (2015) y Diálogos de la forma perdida (2016), ambas traducidas por Carmen Ruiz de Apodaca; la colección de aforismos de Mario Andrea Rigoni, Vanidad (2017), traducida por Fabrizio Cossalter.

La editorial independiente Taller Ditoría publicó una traducción de dominio público del Cuento de Alibech de Giovanni Boccaccio (2009), y de Giacomo Leopardi, Sobre el amor. Antología del Zibaldone (2009), a cargo de Guillermo Fernández.

La colección Autoria, que marca la colaboración de Taller Ditoría con la editorial independiente italiana Auieo, dirigida por el editor y escritor Marco Perilli, publicó, además del ya mencionado Cuento del gordo carpintero, la primera traducción al español, a cargo de Nayelly Zabaleta Solís, de Kn (2009) de Carlo Belli, una colección de aforismos de 1935 que fue definida "el evangelio del arte abstracto" 17 por Vasili Kandinsky.

En 2011 nace la sección mexicana de Auieo, y, además de reeditar en versión bilingüe la traducción de Lamberti de El cuento del gordo carpintero (2015), publica, siempre en versión bilingüe, las traducciones de Guillermo Fernández de Jóvenes sin trabajo de Valerio Magrelli (2011) y de una selección de textos de Leonardo da Vinci no relacionados directamente con sus investigaciones científicas, ni con su faceta pictórica, titulada Bestiario, fábulas y pensamientos (2014). De Magrelli Auieo publicó también la traducción de Marisela Valdés del ensayo Mirar la lengua "desde afuera”: Mallarmé y la traducción (2013).

Además del ya mencionado Pavese, El Tucán de Virginia publicó la traducción de Guillermo Fernández de Cantos órficos de Dino Campana (1990) y Poemas de Valerio Magrelli (1990), y Escúchame señor de Emilio Coco, traducido por Marco Antonio Campos (2013).

Para las Ediciones Papeles Privados, la poeta y traductora Elvia De Angelis publicó la poesía completa de Cesare Pavese (2001).

Para su propia editorial, Vaso Roto, domiciliada en España (Madrid) y en México (San Pedro Garza García, N. L.), que se especializa sobre todo en poesía, la escritora y traductora de poesía Jeannette L. Clariond ha publicado sus traducciones de tres poemarios de Alda Merini: Cuerpo de amor (2009), Magnificat (2009) y La carne de los ángeles (2009), además de Francisco. Canto de una criatura (edición bilingüe;

\footnotetext{
${ }^{17}$ Encontramos esta definición en el catálogo de la muestra La Vanguardia Aplicada 1890-1950. Madrid, Fundación Juan March, 2012: 97, donde se cita como fuente, en la nota 113, la primera edición de $K n$, de 1935, sin especificar números de página, por lo que dedujimos que se encuentra en la contraportada.
} 
2014) y la selección Cuerpo del dolor (2017). Para la editorial mexicana Papeles Privados, Clariond tradujo una Antología de Roberto Carifi (2000); para editoriales españolas, de Merini La tierra Santa (Valencia, Pre-textos, 2001) y Baladas no pagadas (2005, Barcelona, Editorial La Poesía, Señor Hidalgo), y de Primo Levi A una hora incierta (2005, Barcelona, Editorial La Poesía, Señor Hidalgo).

Para Vaso Roto, Eduardo Montagner Anguiano, que proviene de la comunidad de origen véneta asentada en Chipilo, y Giampiero Bucci Gabrielle, académico de origen romano, trabajaron juntos para traducir un volumen de poesía de Andrea Zanzotto: $E l$ (necesario) mentir. Prosa selecta. (2012).

Finalmente mencionamos una serie de traducciones, publicadas por editoriales independientes, que se relacionan muy directamente con el Departamento de Letras Italianas y con la Cátedra Extraordinaria Italo Calvino. Dies Irae (Garabatos, 2015) de Antonio de Petro, con la traducción de Víctor García Salas, egresado de nuestra licenciatura con una tesis que analiza esta novela, y a cargo también, junto con Carlos Ciade Castellanos, de la traducción de las Poesías de Giovanni Riva (Ediciones del Lirio, 2015). Ambos trabajos surgen y se realizaron gracias a los seminarios que por muchos años impartió Paola Leoni en nuestra Facultad, quien además contribuyó con algunas de sus ponencias a la difusión y al análisis tanto de estos dos autores como de una constelación muy rica de la literatura italiana del siglo XX.

\section{Obras citadas}

AmARA, Luigi. 2015. "Una hidra de seis cabezas. Tumbona Ediciones". Itinerarios de la cultura contemporánea en México. Ed. Benjamín MAYER FoulKeS. México: Conaculta / 17, Instituto de Estudios Críticos. Pp. 65-74.

DeBenedeTti, Giacomo. 2003. Il romanzo del Novecento. Milán: Garzanti.

Escalante Gonzalbo, Fernando. 2015. "El interés público”. Ed. Benjamín Mayer Foulkes. México: Conaculta / 17, Instituto de Estudios Críticos. Pp. 75-84.

La Vanguardia Aplicada 1890-1950. 2012. [Catálogo de exposición. Fundación Juan March, Madrid]. Madrid: Fundación Juan March.

LARA ZaVAla, Hernán. 2005. "La Cátedra Italo Calvino". Italo Calvino, homenajes y análisis. Ed. María CARRILLO. México: UNAM, Cátedra Extraordinaria Italo Calvino. Pp. 21-22.

MazzUCATO, Mariana. 2013. El Estado emprendedor. Barcelona: RBA Libros.

MorÁBito, Fabio. 2001. "Prólogo". Torquato TAsso, Aminta. México: UnAM. Pp. 9-12.

RABASA, Eduardo. 2015. “¿De verdad estoy aquí bromeando con Magris? Sexto Piso". Itinerarios de la cultura contemporánea en México. Ed. Benjamín Mayer Foulkes. México: Conaculta / 17, Instituto de Estudios Críticos. Pp. 217-222. 
SALVADOR, Mauricio. 2015. "Llenar un vacío de crítica. Hermano cerdo". Itinerarios de la cultura contemporánea en México. Ed. Benjamín MAYER FoulKeS. México: Conaculta / 17, Instituto de Estudios Críticos. Pp. 211-216.

Serrano Coronado, Tomás y Céline Desmet, coord. 2011. Así va el cuento... Antología de traducción. México: UnAM, Coordinación de Humanidades, Centro de Enseñanza de Lenguas Extranjeras.

Villarreal, Rogelio. 2015. "Abatir el concepto mismo de revista cultural". Itinerarios de la cultura contemporánea en México. Ed. Benjamín MAYER FoulKes. México: Conaculta / 17, Instituto de Estudios Críticos. Pp. 203-210. 